\title{
Subjective cognitive functioning in relation to changes in levels of depression and anxiety in youth over 3 months of treatment
}

Kelly Allott, Caroline Gao, Sarah E. Hetrick, Kate M. Filia, Jana M. Menssink, Caroline Fisher, Ian B. Hickie, Helen E. Herrman, Debra J. Rickwood, Alexandra G. Parker, Patrick D. Mcgorry and Sue M. Cotton

\section{Background}

Subjective cognitive difficulties are common in mental illness and have a negative impact on role functioning. Little is understood about subjective cognition and the longitudinal relationship with depression and anxiety symptoms in young people.

\section{Aims}

To examine the relationship between changes in levels of depression and anxiety and changes in subjective cognitive functioning over 3 months in help-seeking youth.

\section{Method}

This was a cohort study of 656 youth aged $12-25$ years attending Australian headspace primary mental health services. Subjective changes in cognitive functioning (rated as better, same, worse) reported after 3 months of treatment was assessed using the Neuropsychological Symptom Self-Report. Multivariate multinomial logistic regression analysis was conducted to evaluate the impact of baseline levels of and changes in depression (nineitem Patient Health Questionnaire; PHQ9) and anxiety symptoms (seven-item Generalised Anxiety Disorder scale; GAD7) on changes in subjective cognitive function at follow-up while controlling for covariates.

\section{Results}

With a one-point reduction in PHQ9 at follow-up, there was an estimated $11-18 \%$ increase in ratings of better subjective cognitive functioning at follow-up, relative to stable cognitive functioning. A one-point increase in $\mathrm{PHQ}$ from baseline to follow-up was associated with $7-14 \%$ increase in ratings of worse subjective cognitive functioning over 3 months, relative to stable cognitive functioning. A similar attenuated pattern of findings was observed for the GAD7.

\section{Conclusions}

A clear association exists between subjective cognitive functioning outcomes and changes in self-reported severity of affective symptoms in young people over the first 3 months of treatment. Understanding the timing and mechanisms of these associations is needed to tailor treatment.

\section{Keywords}

Subjective cognitive functioning; depression; anxiety; youth; longitudinal.

\section{Copyright and usage}

(c) The Author(s) 2020. This is an Open Access article, distributed under the terms of the Creative Commons Attribution licence (http://creativecommons.org/licenses/by/4.0/), which permits unrestricted re-use, distribution, and reproduction in any medium, provided the original work is properly cited.

\section{Background}

Subjective cognitive difficulties, complaints or failures (such as losing one's train of thought, forgetting important information or having trouble concentrating) are commonly experienced by people with mental disorders. ${ }^{1-3}$ Subjective cognitive difficulties are not significantly correlated with objective clinician-administered tests of cognitive performance, ${ }^{4-9}$ but are significantly associated with a range of individual factors, including levels of stress, chronotype, substance use, sleep quality and mental health symptoms to name a few. ${ }^{2,5,6,10-13}$ People with depression and anxiety disorders appear especially susceptible to subjective cognitive difficulties; ${ }^{1-3,9,14,15}$ these difficulties are associated with severity of affective symptoms ${ }^{16-19}$ and have a negative impact on role func-

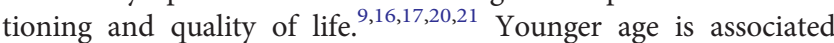
with higher levels of subjective cognitive difficulties in people with depression. ${ }^{3,9,17}$ Together, these findings suggest that subjective cognitive functioning is important to consider independently of objective cognition in research, clinical formulation and treatment.

To date, most studies have been cross-sectional and limited to specific diagnostic groups. However, affective symptoms are common to all mental health conditions and little is known about the temporal relationship between subjective cognitive functioning and affective symptoms cross-diagnostically. It is important to understand how subjective cognitive symptoms relate to affective symptoms early in the course of mental illness and over time, given they are shown to be more strongly associated with self-efficacy and functioning than objective cognitive difficulties. ${ }^{22}$ Ongoing subjective cognitive complaints may increase one's risk for maintenance of affective symptoms, relapse or poor functional outcomes. ${ }^{20,23,24}$ Similarly, improvements in subjective cognitive functioning may signal clinical and functional improvement. Subjective cognitive functioning can be easily assessed within clinical settings and thus, research understanding its course and clinical significance remains an important endeavour.

\section{Aims}

The aim of this study was to examine the relationship between changes in levels of depression and anxiety, and changes in subjective cognitive function over the first 3 months of treatment in a large sample of youth aged 12-25 attending primary care mental health services (headspace) in Australia. We hypothesised that improvements or worsening in anxiety and depression symptoms over 3 months would be associated with subjective improvements or worsening in cognitive functioning, respectively, while controlling for demographic factors and substance use.

\section{Method}

\section{Design, setting and participants}

This study involved analysis of baseline and 3-month follow-up data from a cohort study examining characteristics and outcomes of 
young people presenting for mental health treatment: the Comprehensive Outcome Measurement for Youth (Y-COM) study. Participants were recruited from three metropolitan and two regional headspace centres across Australia. headspace was established in 2006 and is Australia's federally funded primary care service that provides highly accessible evidence-based early intervention and integrated support to people aged 12-25 years experiencing, or at risk of developing, mental or substance use disorders. ${ }^{25-27}$ Centres have a number of health professionals offering services at no or low-cost under the Federal Government's Medicare Benefits Schedule. Each multidisciplinary team has various combinations of general practitioners, psychiatrists, allied health professionals (for example psychologists, social workers, nurses, occupational therapists), vocational specialists, and intake and assessment clinicians comprising an 'access team'. The headspace model typically involves an engagement and assessment session with the Access team to determine the person's presenting concerns and needs. The young person is then referred to the appropriate service provider/clinician(s) for treatment. Young people aged 12-25 years (inclusive) presenting to the study headspace centres for the first appointment with concerns about mental health or substance use-related problems were considered eligible for the study. There was no emphasis on self-reported mood or cognitive symptoms for study inclusion.

\section{Measures}

A larger battery of self-report and clinician-rated measures were administered at baseline and follow-up in the primary Y-COM study; only those relevant to the current study are reported here. Demographic characteristics of relevance to this analysis included age and gender assigned at birth. Primary diagnoses based on the DSM $-5^{28}$ were determined by participants' clinicians.

\section{Depression and anxiety}

Depressive symptoms were measured using the Patient Health Questionnaire (PHQ9), ${ }^{29}$ a nine-item self-report instrument for measuring depression severity over the previous two weeks. All items are rated on a four-point scale from 0 (not at all) to 3 (nearly every day), providing a 0 to 27 severity score. PHQ9 scores of 5,10 , 15 and 20 were taken as the cut-off points for mild, moderate, moderately severe and severe depression, respectively. ${ }^{29}$ Anxiety symptoms were measured using the Generalised Anxiety Disorder scale (GAD7), ${ }^{30}$ a seven-item self-report instrument for measuring symptoms of anxiety over the previous 2 weeks. All items are rated on a four-point scale from 0 (not at all) to 3 (nearly every day), providing a 0 to 21 severity score. GAD7 scores of 5,10 and 15 were taken as the cut-off points for mild, moderate and severe anxiety, respectively. ${ }^{30}$

\section{Subjective cognitive functioning}

Subjective cognitive functioning was assessed at 3-month follow-up using the Neuropsychological Symptom Self-Report (NSSR). ${ }^{14}$ The NSSR is a brief eight-item self-report questionnaire designed to assess an individual's subjective changes in cognitive functioning (for example thinking speed, memory, concentration) since commencing treatment. For each item, on a three-point scale the participant indicates whether their cognitive functioning is (a) better than, (b) the same, or (c) worse than before they started treatment; for the current study, this was over the previous 3 months.

\section{Covariates: substance use}

Substance use was assessed using the World Health Organization Alcohol, Smoking and Substance Involvement Screening Test (WHO-ASSIST).$^{31}$ In the current study we focused on alcohol and cannabis use as these are the substances most commonly used by young people that may have an impact on cognition. ${ }^{4}$ We defined moderate-to-high alcohol use as a score of 11 and over and moderate-to-high cannabis use as a score of 4 and over in accordance with the WHO-ASSIST guidelines. ${ }^{31}$

\section{Procedure}

Recruitment for the study occurred from September 2016 to April 2018. Research assistants were embedded within the headspace Access teams to identify eligible study participants. Advertisements were also placed in the centre waiting rooms to increase visibility of the study among potential participants. Written informed consent was obtained from all participants and a parent/guardian for participants aged $<18$ years. Following consent, participants completed a battery of self-report measures via tablet computers under the guidance of a research assistant. Participants were contacted 3 months post-baseline to complete the follow-up assessment. Participants were reimbursed AU\$30 per assessment (baseline and follow-up).

All procedures contributing to this work complied with the ethical standards of the relevant national and institutional committees on human experimentation and with the Helsinki Declaration of 1975, as revised in 2008. All procedures were reviewed and approved by the University of Melbourne Human Research Ethics Committee, and the local Human Ethics and Advisory Group (1645367.1).

\section{Statistical analysis}

Analyses were conducted using R version 3.6.1. Descriptive statistics such as means (s.d.) and percentages (counts) were used to characterise the cohort. Internal consistency between NSSR items was evaluated using Cronbach's alpha. Multivariate multinomial logistic regression analyses were conducted to evaluate the impact of baseline levels of and changes in anxiety and depression symptoms on different aspects of subjective cognitive function at follow-up, while controlling for other key confounding factors including age, gender, diagnosis, alcohol and cannabis use. Ordinal logistic regression models were not applied because of violation of proportional odds assumption in the data. Because of an issue of multicollinearity between GAD7 and PHQ9, their effects were examined in separate models.

Results from the multinomial logistic regression analyses are presented as relative risk ratios (RRRs) with $95 \%$ CIs of subjective improvement (better than before treatment) or decline (worse than before treatment) in cognitive functioning relative to stable (same as before treatment) cognitive functioning that is associated with each unit change in the predictor (for example for each unit increase in PHQ9 score reduction). The level of statistical significance was set at $P<0.05$ (two-sided). To obtain more accurate estimates, and to control for non-response bias, multiple imputation using chained equations were incorporated in the analysis to address missing data for both the outcome and predictor variables. ${ }^{32}$ Data were imputed for participants who had incomplete data $(n=23)$. Sensitivity analyses were conducted with unimputed data as well as using standardised scores instead of crude scores.

\section{Results}

\section{Consent and participant flow}

As shown in Fig. 1, there were 2126 youth across the five headspace services who were invited to participate in the study. Of the 1019 youth who were excluded, many declined to participate $(52.9 \%$, $n=539$ ) or consent could not be obtained because of non-response to being invited or non-attendance at scheduled consenting appointments $(43.2 \%, n=440)$. In terms of participation, a vast majority of consented $(n=1144)$ participants $(96.8 \%, n=1107)$ completed the baseline questionnaire battery. 


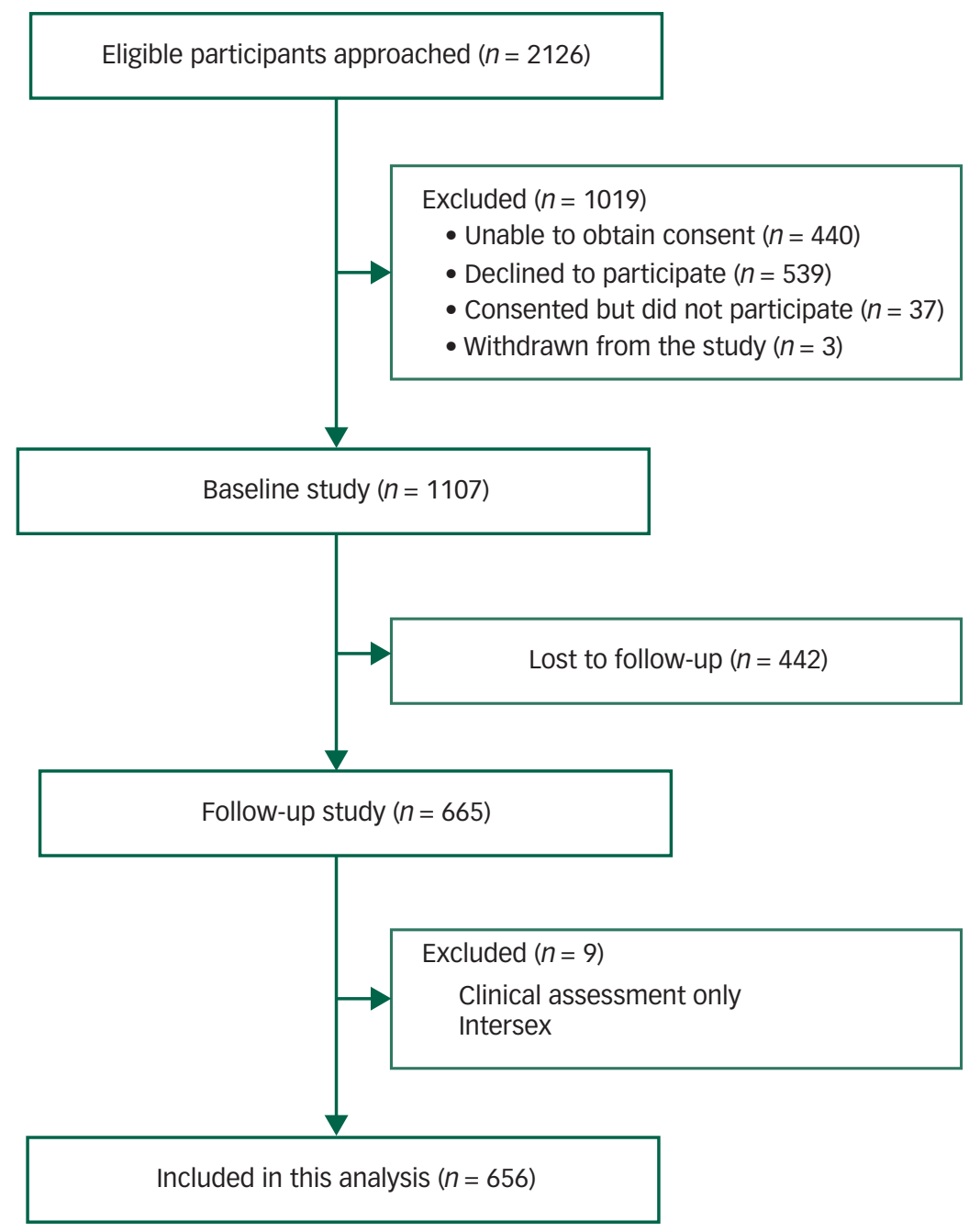

Fig. 1 Participant flow diagram.

Of those with baseline data, $60.0 \%(n=665)$ had follow-up data at 3 months. Of these, a further nine participants were excluded because they did not complete any self-report questionnaires at follow-up or reported being intersex (numbers were too small to be included as a separate group in the analysis). Based on the baseline data, neither demographic characteristics (age and gender) nor clinical factors (diagnosis, anxiety and depression symptoms) were found related to the chance of being lost to follow-up. A total of 656 participants were included in the current analysis.

\section{Participant characteristics}

Table 1 shows that participant age was distributed fairly evenly between the ages of 12 and 25 years, with a slightly lower proportion of participants aged between 12 and 14 years. The mean age of the group was 18.2 (s.d. = 3.3). More participants were female (66.5\%). The most frequently found primary diagnoses were anxiety and/or depression (75.9\%). A total of $16 \%$ of participants reported moderate or high levels of alcohol use and $17 \%$ reported moderate or high levels of cannabis use.

\section{Depression and anxiety}

A substantial proportion of the participants reported high levels of anxiety and depression symptoms. At baseline, the mean PHQ9 score was 12.8 (s.d. $=6.5$ ) and $66.9 \%$ had moderate-to-severe depression (see Table 2). The mean PHQ9 symptom score was reduced to 9.8 (s.d. $=6.5$ ) at 3 -month follow-up with an average reduction of 3 points (s.d. $=6$ ) and the proportion of participants in the moderate-to-severe depression range was reduced to $46.9 \%$.

\section{Table 1 Participants' characteristics ${ }^{a}$}

\begin{tabular}{|c|c|}
\hline Age, mean (s.d.) & $18.2(3.3)$ \\
\hline \multicolumn{2}{|l|}{ Age group, years: $n$ (\%) } \\
\hline $12-14$ & $100(15.2)$ \\
\hline $15-17$ & $190(29.0)$ \\
\hline $18-20$ & $196(29.9)$ \\
\hline $21-25$ & $170(25.9)$ \\
\hline Female, $n(\%)$ & $436(66.5)$ \\
\hline \multicolumn{2}{|l|}{ Primary diagnosis, ${ }^{\mathrm{b}} \mathrm{n}(\%)$} \\
\hline Depression & $110(17.4)$ \\
\hline Anxiety & $182(28.8)$ \\
\hline Depression and Anxiety & $188(29.7)$ \\
\hline Other ${ }^{c}$ & $152(24.1)$ \\
\hline Moderate or high alcohol use, $n$ (\%) & $100(16.2)$ \\
\hline Moderate or high cannabis use, $n$ (\%) & $106(17.0)$ \\
\hline
\end{tabular}

a. Missing data include: 24 participants for primary diagnosis, 40 for alcohol use risk and 34 for cannabis use risk.

b. Primary diagnosis was determined by the participants' clinicians based on DSM-5. c. The other diagnoses were as follows: psychosis $2(0.3 \%)$; substance use $9(1.4 \%)$; behavioural disorder 9 (1.4\%); personality disorder $19(3.0 \%)$; adjustment disorder 7 (1.1\%); provisional diagnosis $15(2.4 \%)$; other $91(14.4 \%)$. 
Table 2 Participants' anxiety and depression symptoms

\begin{tabular}{|c|c|c|}
\hline & $\begin{array}{l}\text { Baseline } \\
(n=656)\end{array}$ & $\begin{array}{l}\text { Follow-up } \\
(n=656)\end{array}$ \\
\hline \multicolumn{3}{|l|}{ Depression severity measured by PHQ9 } \\
\hline Score, mean (s.d.) & $12.8(6.5)$ & $9.8(6.5)$ \\
\hline \multicolumn{3}{|l|}{ PHQ9 risk category, $n$ (\%) } \\
\hline Minimal depression (1-4) & 79 (12.2) & $152(23.8)$ \\
\hline Mild depression (5-9) & $136(20.9)$ & $187(29.3)$ \\
\hline Moderate depression (10-14) & $172(26.5)$ & $138(21.6)$ \\
\hline Moderately severe depression (15-19) & 149 (22.9) & $107(16.7)$ \\
\hline Severe depression (20-27) & $114(17.5)$ & $55(8.6)$ \\
\hline PHQ9 score reduction from baseline, mean (s.d.) & & $-3.0(-6.0)$ \\
\hline \multicolumn{3}{|l|}{ Anxiety severity measured by GAD7 } \\
\hline Score, mean (s.d.) & $10.2(5.6)$ & $7.9(5.5)$ \\
\hline \multicolumn{3}{|l|}{ GAD7 risk category, $n$ (\%) } \\
\hline Minimal anxiety $(0-4)$ & $115(17.6)$ & $189(29.4)$ \\
\hline Mild anxiety (5-9) & $188(28.8)$ & $220(34.3)$ \\
\hline Moderate anxiety (10-14) & $186(28.5)$ & $156(24.3)$ \\
\hline Severe anxiety (15-21) & $163(25.0)$ & $77(12.0)$ \\
\hline GAD7 score reduction from baseline, mean (s.d.) & & $-2.3(-5.3)$ \\
\hline \multicolumn{3}{|l|}{ Correlation coefficients } \\
\hline Between PHQ9 and GAD7 & 0.67 & 0.69 \\
\hline Between change of PHQ9 and change of GAD7 & & 0.58 \\
\hline \multicolumn{3}{|c|}{$\begin{array}{l}\text { PHQ9, nine-item Patient Health Questionnaire; GAD7, seven-item Generalised Anxiety } \\
\text { Disorder scale. } \\
\text { a. Missing data were excluded: } 6 \text { records for PHQ9 at baseline, } 17 \text { records for PHQ9 at } \\
\text { follow-up, } 22 \text { records for change of PHQ9 from baseline, } 4 \text { records for GAD7 at baseline, } \\
14 \text { records for GAD7 at follow-up and } 16 \text { records for change of GAD7 from baseline. }\end{array}$} \\
\hline
\end{tabular}

Similarly, the mean GAD7 score at baseline was 10.2 (s.d. = 5.6), which was reduced to a mean score of 7.9 (s.d. $=5.5$ ) at follow-up, with a mean reduction of 2.3 (s.d. $=5.3$ ). The proportion within the range of moderate-to-severe anxiety reduced from $53.5 \%$ at baseline to $36.3 \%$ at follow-up. Large positive correlations were observed between PHQ9 and GAD7 at both baseline and followup (both $r>0.60$ ).

\section{Subjective cognitive function at follow-up}

Across each item of the NSSR, most participants reported that their subjective cognition was the same as before starting treatment (see Table 3). About a quarter to a third of participants reported subjective improvement in various aspects of cognitive functioning at 3-month follow-up after treatment. The percentage of participants who reported improvement in their ability to remember verbal instructions and conversations was slightly lower (17.2\%) compared with other subjective cognitive symptoms. A small percentage of participants $(<10 \%)$ reported experiencing subjective deterioration in cognitive functioning since commencing treatment. Internal consistency (Cronbach's alpha based on polychoric correlation coefficients) for the NSSR was 0.93 for the cohort.

\section{Association between subjective cognitive function and symptoms of anxiety and depression}

A clear association was observed between subjective cognitive function outcomes (NSSR) and changes in self-reported severity of anxiety (measured by GAD7) and depression (measured by PHQ9) symptoms (Supplementary Figs 1 and 2 available at https://doi.org/10.1192/bjo.2020.68).

Results from multinomial logistic regression models are displayed in Fig. 2. When controlling for baseline PHQ9 scores and other confounding factors, with one-point reduction in PHQ9 from baseline to follow-up, there was an estimated $11-18 \%$ increase in rates of reporting better cognitive functioning over the 3 months for different NSSR outcomes. One-point increase of PHQ9 from baseline to follow-up was associated
Table 3 Participants' Neuropsychological Symptom Self-Report (NSSR) outcomes $^{\mathrm{a}}$

Participants

$(n=656)$

The speed of completing activities

Worse than before treatment $61(9.6)$

Same as before treatment $407(64.3)$

Better than before treatment

Ability to pay attention and concentrate

Worse than before treatment

$165(26.1)$

same as before treatment

Better than before treatment $176(27.8)$

speed of thinking

Worse than before treatment $42(6.6)$

Same as before treatment 451 (71.2)

Better than before treatment $140(22.1)$

Ability to remember verbal instructions and conversations

Worse than before treatment 62 (9.8)

Same as before treatment $\quad 462$ (73.0)

Better than before treatment 109 (17.2)

Ability to think of words and get words out when speaking

Worse than before treatment

$61(9.6)$

Same as before treatment $400(63.2)$

Better than before treatment

Ability to plan ahead and organise things

Worse than before treatment

$172(27.2)$

Same as before treatment

Better than before treatment

$37(5.8)$

$399(63.0)$

197 (31.1)

Ability to think about more than one thing at a time

Worse than before treatment

33 (5.2)

$432(68.2)$

$168(26.5)$

Motivation to do my usual activities

Worse than before treatment

$64(10.1)$

Same as before treatment

$323(51.0)$

Better than before treatment

$246(38.9)$

a. A total of 23 out of 656 participants did not complete the NSSR questions.

with a $7-14 \%$ increase in rates of reporting worsening of cognitive functioning for different NSSR outcomes (see Fig. 2a). Baseline PHQ9 scores were also associated with change in subjective cognitive functioning over 3 months. For those with the same level of change in PHQ9 scores, a higher baseline PHQ9 score was associated with higher chance of deterioration in subjective cognitive functioning, and a lower baseline PHQ9 score was associated with a higher chance of subjective cognitive improvement.

Similar to depression symptoms, associations between baseline and changes in anxiety symptoms and subjective cognitive functioning were also identified. For a one-point reduction in GAD7 scores from baseline to follow-up, there was an estimated 11-13\% increased rates of better subjective cognitive functioning for different NSSR outcomes (see Fig. 2b). Increase in GAD7 scores from baseline to follow-up was associated with deterioration of most aspects of subjective cognitive functioning except for 'ability to plan ahead and organise things' and 'the speed of completing activities'.

As with depression, a higher baseline GAD7 score was found to be associated with a higher chance of deterioration in subjective cognitive functioning when controlling for changes in GAD7 scores and other confounding factors. In contrast to depression, there was no evidence of lower baseline GAD7 score being associated with a higher chance of improvement in subjective cognitive functioning. Detailed results from multinomial logistic models are provided in Supplementary Tables 1-8 and models with standardised baseline and change scores of PHQ9 and GAD7 are provided in Supplementary Fig. 3. 
(a) The speed of completing activities

Ability to pay attention and concentrate

Speed of thinking

Ability to remember verbal instructions and conversations

Ability to think of words and get words out when speaking

Ability to plan ahead and organise things

Ability to think about more than one thing at a time

Motivation to do my usual activities

(b)

The speed of completing activities

Ability to pay attention and concentrate

Speed of thinking

Ability to remember verbal instructions and conversations

Ability to think of words and get words out when speaking

Ability to plan ahead and organise things

Ability to think about more than one thing at a time

Motivation to do my usual activities
Baseline PHO9

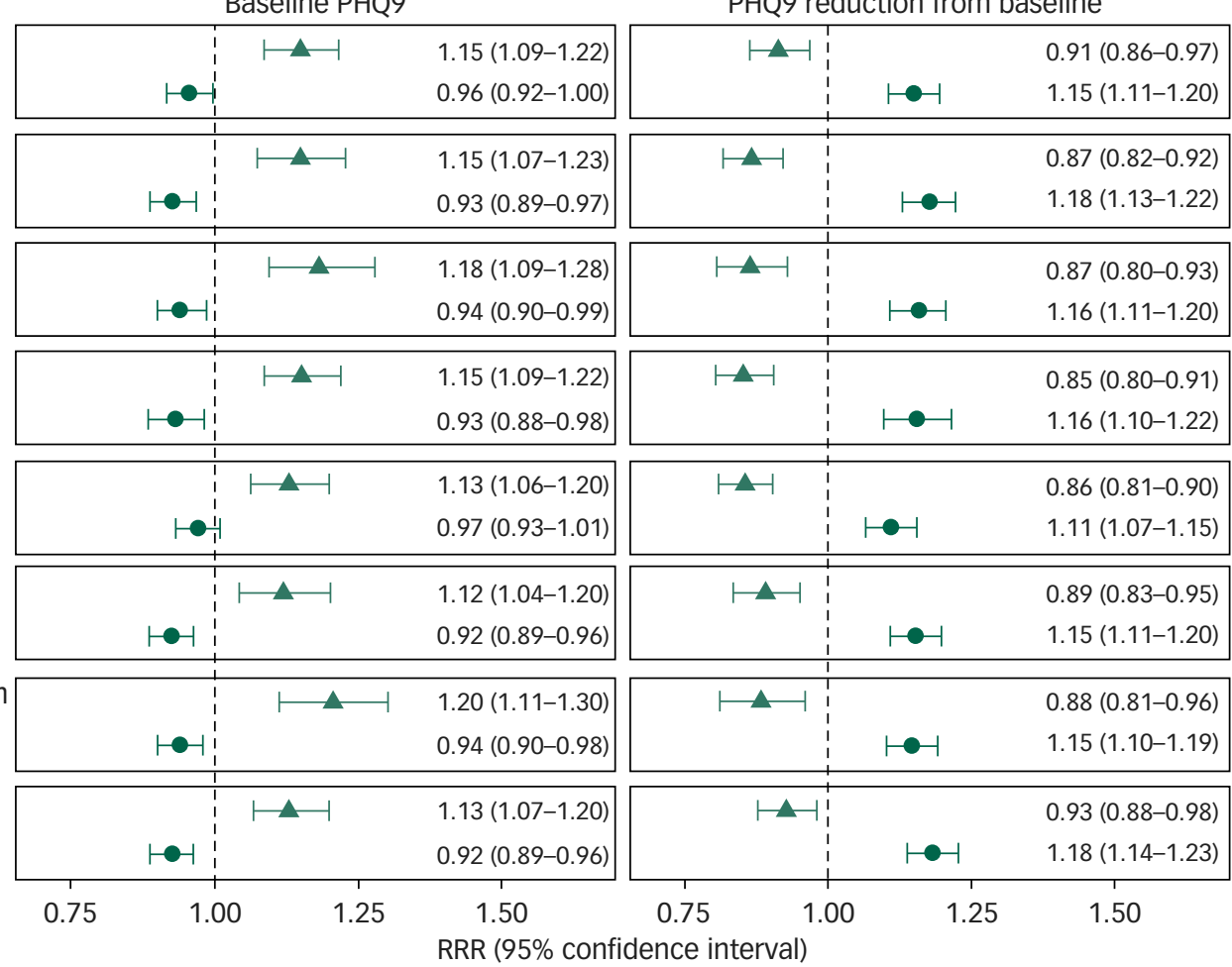

$\rightarrow$ Better than before treatment $\_$Worse than before treatment 


\section{Discussion}

\section{Main findings}

To our knowledge this is the first study to examine subjective cognitive functioning in relation to longitudinal change in affective symptoms in youth presenting for mental health treatment. On average, the participants reported moderate levels of depression and anxiety symptoms, which reduced to the mild range over 3 months. Although most participants perceived general stability in their cognitive functioning over the 3-month study period, approximately one-third reported perceived general improvement since commencing treatment. It was concerning, however, that 5-10\% of participants perceived a decline in various aspects of their cognitive functioning over the follow-up period.

In support of our hypothesis, the main finding was evidence for a strong association between changes in symptoms of depression and anxiety and changes in subjective cognitive functioning in youth over the first 3 months of mental health treatment. This relationship was the case for every symptom of subjective cognitive functioning assessed when controlling for baseline anxiety and depression symptoms, as well as other key confounders. The estimated effect size associated with changes in symptoms of depression and anxiety were comparable across all cognitive symptoms, which suggests that changes in subjective cognitive functioning in relation to changes in affective symptoms may be a unitary process.

A further finding was that when holding change in symptoms constant, higher or lower baseline levels of depression were significantly associated with perceived decline or improvement in cognitive functioning over 3 months, respectively. For baseline anxiety, higher levels were also associated with a higher chance of deterioration in subjective cognitive functioning, whereas lower baseline anxiety was not associated with a higher chance of improvement in subjective cognitive functioning over 3 months. This suggests that higher severity of affective symptoms at entry to treatment increases one's risk of subjective cognitive decline.

\section{Comparison with findings from other studies}

Our finding of a strong relationship between affective symptoms and subjective cognition are consistent with a large body of crosssectional research conducted in adult samples with affective disorders (for example ${ }^{11,16,17-19}$ ). We extend this work by demonstrating a relationship between affective symptoms and subjective cognitive function in a sample of young people with various mental health concerns and diagnoses, which suggests that the relationship between these variables occurs early in the course of mental illness and is not diagnosis specific. Furthermore, most previous work has focused on depressive symptoms and subjective cognition, but here we have shown that a similar relationship exists with symptoms of anxiety. In support of these findings, previous cross-sectional research both in first-episode psychosis and established schizophrenia showed that depressive and anxiety symptoms were the strongest predictors of subjective cognitive functioning, after accounting for objective cognitive functioning, medication, positive and negative symptoms. ${ }^{5,33}$

\section{Interpretation of our findings}

The findings of the current study support the notion that subjective cognitive impairment is at least in part a state-related phenomenon. ${ }^{34}$ However, because we measured subjective change in cognitive functioning only at the follow-up time point, we were unable to determine what level the participants perceived their cognitive functioning to be at service entry (baseline). This is especially relevant for the largest group who perceived no change in their cognitive functioning over 3 months. It is possible that despite perceived stability, a subgroup of these participants experienced longstanding subjective cognitive impairments. The same could be said for those who perceived improvement in cognitive functioning, where, despite improvement, they still may be performing below their desired level.

Our data do not elucidate the timing of subjective cognitive difficulties and affective symptoms in these young people. Gaining a clearer understanding of the dynamic interplay between these symptoms over time will help inform interventions and clinical recommendations. Temporal relationships may also differ between individuals. For example, subjective cognitive difficulties may be an early manifestation of the clinical expression of anxiety and depressive symptoms, thus interventions designed to identify and address cognitive complaints early may help to promote mental health in young people. Conversely, if anxiety and depressive symptoms tend to precede cognitive concerns, then treatment focused on reducing affective symptoms may be adequate. Future studies should measure subjective cognitive functioning at multiple time points to better characterise the relationship between subjective cognitive functioning and affective symptoms.

The mechanism(s) underpinning the relationship between affective symptoms and subjective cognitive function are not well understood and likely to be multiple and complex. Several pathways may be considered. First, consistent with some of the diagnostic criteria for depression and anxiety disorders, there are items on the self-report measures that ask about cognitive problems. For example, the PHQ9 specifically asks about concentration difficulties. However, this is unlikely to entirely explain the relationship between affective symptoms and subjective cognitive functioning because the NSSR asks about a number of cognitive functions that do not overlap with the PHQ9 or GAD7 and vice versa. Psychological models posit that cognitive biases or maladaptive schemas might contribute to negative appraisal of cognitive function, which may have a further impact on mood, coping strategies and functioning. ${ }^{11,35,36}$

Alternatively, cognitive failures in daily life may increase one's negative affect. ${ }^{23}$ Research has shown that subjective cognitive impairment in mood disorders is associated with poorer socio-occupational functioning, ${ }^{37,38}$ which may in turn have an impact on mood. A vicious cycle may ensue where cognitive difficulties and affective symptoms feed into one another. ${ }^{23,36}$ When subjective cognitive impairment is perceived to have a negative impact on important aspects of daily life, the influence on the development or exacerbation of depression or anxiety symptoms may be greater. It is also possible that depleted cognitive resources are a consequence of poor sleep and mental fatigue, which are common in anxiety and depression. ${ }^{12}$

A further possibility is the depressive realism hypothesis, which suggests that individuals with depression make more realistic and accurate inferences or judgements than healthy persons. ${ }^{39}$ Recent research in fact provides evidence for an underestimation of cognitive ability in people with depression and an overestimation of cognitive ability in healthy controls relative to their objective cognitive performance. ${ }^{40}$ However, the discrepancy between subjective and objective cognitive functioning in individuals with depression may vary as a function of their age and depression severity. ${ }^{8}$ Clearly, more work is needed to understand how these factors relate to one another.

The current study suggests that subjective cognitive functioning (in addition to objective measures) deserves clinical attention, particularly in those who perceive a decline in their cognitive functioning despite having accessed treatment. Ongoing subjective cognitive impairment is commonly found in people who are in partial or full remission from depression. ${ }^{11}$ A 3 -year prospective study of adults 
with depression found that subjectively reported cognitive symptoms were the most prominent symptoms during both the acute and remission phases of depression; participants reported experiencing cognitive problems $44 \%$ of the time while in remission from a depressive episode. ${ }^{41}$ Residual self-reported cognitive symptoms have been shown to increase the odds of depression relapse. ${ }^{24}$ Young people with depression report that subjective cognitive difficulties are distressing and interfere with their role functioning (for example study, work, relationships) ${ }^{23}$ In fact, self-reported cognitive difficulties are shown to mediate as much as $25 \%$ of the impact of depression on patients' role functioning. ${ }^{20}$ Therapeutic interventions that aim to reduce subjective cognitive dysfunction in people who are in remission from depression or anxiety could potentially lead to reduced risk of relapse and enhance their functioning. Recent work has shown that providing psychoeducation to young people about cognitive functioning and strategies for managing cognitive difficulties via a fact-sheet was a simple, acceptable and helpful means for beginning to address cognitive concerns in clinical practice. ${ }^{42}$

\section{Strengths and limitations}

There are several strengths of the current study, including the large number of participants and prospective design - previous studies had small sample sizes and were mostly cross-sectional. To our knowledge, no previous study had included youth (adolescents) in their samples. We were also able to control for potential effects of substance use on subjective cognitive functioning in young people presenting for treatment. In previous research examining subjective cognition and affective symptoms there has generally been a lack of consideration for the potential role of substance use, most likely because of the complexity of clinical presentations.

This study also has some limitations. First, objective cognitive functioning was not measured in the current study, which is known to be compromised in youth early in the course of mental health conditions. ${ }^{43,44}$ Evidence consistently shows that there is a low correlation between subjective and objective cognitive impairment. ${ }^{4-7}$ It is possible that subjective and objective cognitive assessments capture different, but equally relevant, aspects of cognition in youth mental health. Longitudinal studies that measure both subjective and objective cognitive functioning are needed to understand the trajectory and unique role that subjective and objective cognitive impairments have in relation to symptom expression and psychological and role functioning.

Second, we did not have a baseline level of subjective cognitive functioning; the NSSR was only administered at one time point and relies on retrospective recall of cognitive functioning, which may be unreliable. Third, data regarding medication use was not collected and medication is known to have an impact on subjective cognitive functioning in varied and complex ways. Although the headspace model prioritises evidence-based psychological therapies in the first instance for most young people, who are likely to be presenting with depression and anxiety, ${ }^{45}$ it is likely that a proportion of participants were taking medication during the 3-month follow-up period, which may have affected the findings. Fourth, there may have been relevant differences between participants who were excluded and those who participated in the study, which we were unable to measure.

\section{Future directions}

To conclude, we identified a strong association between affective symptoms and subjective cognitive functioning among helpseeking young people attending primary care youth mental health services. We highlighted the clinical implications of evaluating subjective cognitive functioning and severity of depression and anxiety symptoms at entry to mental health services. Further studies are needed to evaluate the psychometric properties of the NSSR, particularly across a diverse population with different types and severity of mental health conditions. Further work is needed to gain more understanding of the longitudinal associations between subjective and objective cognitive functioning and affective symptoms.

Kelly Allott (1D), Orygen, Australia; and Centre for Youth Mental Health, The University of Melbourne, Australia; Caroline Gao, Orygen; Centre for Youth Mental Health, The University of Melbourne; and Department of Epidemiology and Preventive Medicine, School of Public Health and Preventive Medicine, Monash University, Australia; Sarah E. Hetrick, Department of Psychological Medicine, University of Auckland, New Zealand; Kate M. Filia, Orygen; and Centre for Youth Mental Health, The University of Melbourne, Australia; Jana M. Menssink, Orygen; and Centre for Youth Mental Health, The University of Melbourne, Australia; Caroline Fisher, Department of Psychology, Royal Melbourne Hospital, Melbourne Health; and The Melbourne Clinic, Australia; Ian B. Hickie, Brain and Mind Centre, The University of Sydney, Australia;

Helen E. Herrman, Orygen; and Centre for Youth Mental Health, The University of Melbourne, Australia; Debra J. Rickwood, headspace National Youth Mental Health Foundation; and Faculty of Health, University of Canberra, Australia;

Alexandra G. Parker, Orygen; Centre for Youth Mental Health, The University of Melbourne; and Institute for Health and Sport, Victoria University, Australia;

Patrick D. Mcgorry, Orygen; and Centre for Youth Mental Health, The University of Melbourne, Australia; Sue M. Cotton, Orygen; and Centre for Youth Mental Health, The University of Melbourne, Australia

Correspondence: Kelly Allott. Email: kelly.allott@orygen.org.au

First received 11 Mar 2020, final revision 26 Jun 2020, accepted 1 Jul 2020

\section{Supplementary material}

Supplementary material is available online at http://doi.org/10.1192/bjo.2020.68.

\section{Data availability}

The data that support the findings of this study are available upon reasonable request and pending additional ethical approval, from the senior author, S.M.C. (sue.cotton@orygen.org.au).

\section{Acknowledgements}

The study was supported by a National Health and Medical Research Council (NHMRC) Partnership Grant (APP1076940). This was a joint project between Orygen, The University of Melbourne and headspace National Youth Mental Health Foundation. K.A. is supported by a Career Development Fellowship from the NHMRC (APP1141207). We thank Dr Sharnel Perera for her contributions in many aspects of the project and the team of research assistants involved in collecting the data. We thank the headspace centre staff and young people whose participation made this study possible.

\section{Author contributions}

All authors contributed to the conception of the study design or the acquisition, analysis and interpretation of the data and approved the final draft of the manuscript. K.A. led the study conceptualisation and co-wrote the first draft of the manuscript. C.G. co-wrote the first draft of the manuscript and conducted the statistical analysis. S.M.C. assisted with the analysis and assisted with the final draft of the manuscript. S.E.H., K.M.F., J.M.M., C.F., I.B.H., H.E.H., D.J.R., A.G.P. and P.D.M. assisted with the final draft of the manuscript.

\section{Declaration of interest}

K.M.F. reports grants from the National Health and Medical Research Council during the cond.M.F. reports grants from the National frealth and Medical Research Council during the conduct of the study. I.B.H. reports grants from Innowell Pty Ltd, grants from NHMRC, grants from
NHMRC, outside the submitted work; and I.B.H. was an inaugural Commissioner on Australia's National Mental Health Commission (2012-18). He is the Co-Director, Health and Policy at the Brain and Mind Centre (BMC), University of Sydney. The BMC operates an early-intervention youth service at Camperdown under contract to headspace. He is the Chief Scientific Advisor to, and a $5 \%$ equity shareholder in, InnoWell Pty Ltd. InnoWell was formed by the University of Sydney (45\% equity) and PWC (Australia; 45\% equity) to deliver the $\$ 30 \mathrm{M}$ Australian Government-funded Project Synergy (2017-20; a 3-year programme for the transformation of mental health services) and to lead transformation of mental health services internationally through the use of innovative technologies. All other authors report no conflict of interest.

ICMJE forms are in the supplementary material, available online at https://doi.org/10.1192/ bjo.2020.68.

\section{References}

1 van den Bosch RJ, Rombouts RP, van Asma MJ. Subjective cognitive dysfunction in schizophrenic and depressed patients. Compr Psychiatry 1993; 34: $130-6$. 
2 Moritz S, Kuelz AK, Jacobsen D, Kloss M, Fricke S. Severity of subjective cognitive impairment in patients with obsessive-compulsive disorder and depression. J Anxiety Disord 2006; 20: 427-43.

3 Srisurapanont M, Mok YM, Yang YK, Chan HN, Della CD, Zainal NZ, et al. Cognitive complaints and predictors of perceived cognitive dysfunction in adults with major depressive disorder: findings from the Cognitive Dysfunction in Asians with Depression (CogDAD) study. J Affect Disord 2018; 232: 237-42.

4 Carrigan N, Barkus E. A systematic review of the relationship between psychological disorders or substance use and self-reported cognitive failures. Cogn Neuropsychiatry 2016; 21: 539-64.

5 Sellwood W, Morrison AP, Beck R, Heffernan S, Law H, Bentall RP. Subjective cognitive complaints in schizophrenia: relation to antipsychotic medication dose, actual cognitive performance, insight and symptoms. PLOS One 2013; 8: e83774.

6 Tong ACY, Chang WC, Chan ANY, Lin JJ. Objective and subjective cognitive functioning in relation to psychopathology among women with early psychosis. Early Interv Psychiatry 2019; 13: 1227-35.

7 Moritz S, Ferahli S, Naber D. Memory and attention performance in psychiatric patients: lack of correspondence between clinician-rated and patient-rated functioning with neuropsychological test results. J Int Neuropsychol Soc 2004; 10: 623-33.

8 Srisurapanont M, Suttajit S, Eurviriyanukul K, Varnado P. Discrepancy between objective and subjective cognition in adults with major depressive disorder. SC Rep 2017; 7: 3901

9 Petersen JZ, Porter RJ, Miskowiak KW. Clinical characteristics associated with the discrepancy between subjective and objective cognitive impairment in depression. J Affect Disord 2019; 246: 763-74.

10 Koyanagi A, Stubbs B, Lara E, Veronese N, Vancampfort D, Smith L, et al Psychotic experiences and subjective cognitive complaints among 224842 people in 48 low- and middle-income countries. Epidemiol Psychiatr SC 2018; 29: e11.

11 Miskowiak K, Vinberg M, Christensen EM, Kessing LV. Is there a difference in subjective experience of cognitive function in patients with unipolar disorder versus bipolar disorder? Nord Psykiatr Tidsskr 2012; 66: 389-95.

12 Cha DS, Carmona N, Cha RH, Zhou AJ, Subramaniapillai M, Mansur RB, et al. Perceived sleep quality predicts cognitive function in adults with major depressive disorder independent of depression severity. Ann Clin Psychiatry 2019; 31 17-26.

13 Coleman MY, Cain SW. Eveningness is associated with greater subjective cognitive impairment in individuals with self-reported symptoms of unipola depression. J Affect Disord 2019; 256: 404-15.

14 Fisher CA, Goodall J, Simmons MB, Allott K, Hetrick SE. Subjective ratings of neurocognitive functioning in depressed young people undergoing treatment: utility of a brief screening tool. Early Interv Psychiatry 2016; 10: 252-7.

15 Cha DS, Carmona NE, Rodrigues NB, Mansur RB, Lee Y, Subramaniapillai M, et al. Cognitive impairment as measured by the THINC-integrated tool (THINC-it): the association with self-reported anxiety in Major Depressive Disorder. J Affect Disord 2018; 238: 228-32.

16 Sumiyoshi T, Watanabe K, Noto S, Sakamoto S, Moriguchi Y, Tan KHX, et al. Relationship of cognitive impairment with depressive symptoms and psychosocial function in patients with major depressive disorder: cross-sectional analysis of baseline data from PERFORM-J. J Affect Disord 2019; 258: $172-8$.

17 Manit S, Yee Ming M, Yen Kuang Y, Herng-Nieng C, Constantine DD, Zuraida ZN et al. Cognitive Dysfunction in Asian Patients with Depression (CogDAD): a cross-sectional study. Clin Pract Epidemol Ment Health 2017; 13: 185-99.

18 Antikainen R, Hanninen T, Honkalampi K, Hintikka J, Koivumaa-Honkanen H, Tanskanen $\mathrm{A}$, et al. Mood improvement reduces memory complaints in depressed patients. Eur Arch Psychiatry Clin Neurosci 2001; 251: 6-11.

19 Serra-Blasco M, Torres IJ, Vicent-Gil M, Goldberg X, Navarra-Ventura G, Aguilar $E$, et al. Discrepancy between objective and subjective cognition in major depressive disorder. Eur Neuropsychopharmacol 2019; 29: 46-56.

20 Buist-Bouwman MA, Ormel J, de Graaf R, de Jonge P, van Sonderen E, Alonso J, et al. Mediators of the association between depression and role functioning. Acta Psychiatr Scand 2008; 118: 451-8.

21 Ott CV, Bjertrup AJ, Jensen JH, Ullum H, Sjaelland R, Purdon SE, et al. Screening for cognitive dysfunction in unipolar depression: validation and evaluation of objective and subjective tools. J Affect Disord 2016; 190: 607-15.

22 Potvin S, Charbonneau G, Juster RP, Purdon S, Tourjman SV. Self-evaluation and objective assessment of cognition in major depression and attention deficit disorder: implications for clinical practice. Compr Psychiatry 2016; 70: $53-64$
23 Morey-Nase C, Phillips LJ, Bryce S, Hetrick S, Wright AL, Caruana E, et al. Subjective experiences of neurocognitive functioning in young people with major depression. BMC Psychiatry 2019; 19: 209

24 Saragoussi D, Touya M, Haro JM, Jonsson B, Knapp M, Botrel B, et al. Factors associated with failure to achieve remission and with relapse after remission in patients with major depressive disorder in the PERFORM study. Neuropsychiatr Dis Treat 2017; 13: 2151-65.

25 McGorry PD, Tanti C, Stokes R, Hickie IB, Carnell K, Littlefield LK, et al. headspace: Australia's National Youth Mental Health Foundation-where young minds come first. Med J Aust 2007; 187 (7 suppl): S68-70.

26 Rickwood DJ, Telford NR, Parker AG, Tanti CJ, McGorry PD. headspace Australia's innovation in youth mental health: who are the clients and why are they presenting? Med J Aust 2014; 200: 108-11.

27 Rickwood D, Paraskakis M, Quin D, Hobbs N, Ryall V, Trethowan J, et al. Australia's innovation in youth mental health care: the headspace centre model. Early Interv Psychiatry 2019; 13: 159-66.

28 American Psychiatric Association. Diagnostic and Statistical Manual of Mental Disorders (5th edn) (DSM-5). American Psychiatric Association, 2013.

29 Kroenke K, Spitzer RL, Williams JB. The PHQ-9: validity of a brief depression severity measure. J Gen Intern Med 2001; 16: 606-13.

30 Spitzer RL, Kroenke K, Williams JB, Löwe B. A brief measure for assessing generalized anxiety disorder: the GAD-7. Arch Intern Med 2006; 166: 1092-7.

31 Humeniuk R, Ali R, Babor TF, Farrell M, Formigoni ML, Jittiwutikarn J, et al. Validation of the alcohol, smoking and substance involvement screening test (ASSIST). Addiction 2008; 103: 1039-47.

32 Buuren S, Groothuis-Oudshoorn K. mice: multivariate imputation by chained equations in R. J Stat Softw 2011; 45: 1-67.

33 Chang WC, Chan TC, Chiu SS, Hui CL, Chan SK, Lee EH, et al. Self-perceived cognitive functioning and its relationship with objective performance in first-episode schizophrenia: the subjective cognitive impairment scale. Compr Psychiatry 2015; 56: 42-50.

34 Allott K, Fisher CA, Amminger GP, Goodall J, Hetrick S. Characterizing neurocognitive impairment in young people with major depression: state, trait, or scar? Brain Behav 2016; 6: e00527.

35 Tandetnik C, Hergueta $T$, Bonnet $P$, Dubois B, Bungener C. Influence of early maladaptive schemas, depression, and anxiety on the intensity of self-reported cognitive complaint in older adults with subjective cognitive decline. Int Psychogeriatr 2017; 29: 1657-67.

36 Farrin L, Hull L, Unwin C, Wykes T, David A. Effects of depressed mood on objective and subjective measures of attention. I Neuropsychiatry Clin Neurosci 2003; 15: 98-104.

37 Jensen JH, Stottrup MM, Nayberg E, Knorr U, Ullum H, Purdon SE, et al. Optimising screening for cognitive dysfunction in bipolar disorder: validation and evaluation of objective and subjective tools. J Affect Disord 2015; 187: 10-9.

38 Demant KM, Vinberg M, Kessing LV, Miskowiak KW. Assessment of subjective and objective cognitive function in bipolar disorder: correlations, predictors and the relation to psychosocial function. Psychiatry Res 2015; 229: 565-71.

39 Moore MT, Fresco DM. Depressive realism: a meta-analytic review. Clin Psychol Rev 2012; 32: 496-509.

40 Schwert C, Stohrer M, Aschenbrenner S, Weisbrod M, Schroder A. Biased neurocognitive self-perception in depressive and in healthy persons. I Affect Disord 2018; 232: 96-102.

41 Conradi HJ, Ormel J, de Jonge P. Presence of individual (residual) symptoms during depressive episodes and periods of remission: a 3-year prospective study. Psychol Med 2011; 41: 1165-74.

42 Bryce S, Cooke M, Yuen HP, Allott K. Acceptability, safety and perceived impact of providing a fact sheet to young people about cognitive symptoms in depression. Early Interv Psychiatry [Epub ahead of print] 11 February 2020. Available from: https://doi.org/10.1111/eip.12945.

43 Goodall J, Fisher CA, Hetrick S, Phillips L, Parrish EM, Allott K. Neurocognitive functioning in depressed young people: a systematic review and meta-anaIysis. Neuropsychol Rev 2018; 28: 216-31.

44 Hermens DF, Naismith SL, Lagopoulos J, Lee RS, Guastella AJ, Scott EM, et al. Neuropsychological profile according to the clinical stage of young persons presenting for mental health care. BMC Psychol 2013; 1: 8.

45 Rickwood DJ, Mazzer KR, Telford NR, Parker AG, Tanti CJ, McGorry PD. Changes in psychological distress and psychosocial functioning in young people visiting headspace centres for mental health problems. Med J Aust 2015; 202: 537-42.
EXTRA

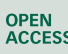

